\title{
Do patients contact their primary care providers before coming to the ER?
}

Kaleb Ware ${ }^{1}$, Josey Noell ${ }^{2}$, Layla Wisser ${ }^{3}$, Katelyn Ware ${ }^{4}$, Peter Pang MD ${ }^{5}$

${ }^{1}$ Indiana University School of Medicine, ${ }^{2}$ Butler University, ${ }^{3}$ Butler University, ${ }^{4}$ Grand Valley State University, ${ }^{5}$ Indiana University School of Medicine, Department of Emergency Medicine

\section{Background and Hypothesis:}

High emergency room (ER) use is considered a healthcare failure. Societal and healthcare structural hurdles are blamed for high ER utilization. One proposed solution is improving healthcare accessability; by having a primary care physician (PCP). With a PCP, patients may avoid utilizing the ER. Whether patients, who have a PCP, attempted to contact their PCP is not well known. Our objective: do patients who self-report having a PCP report attempting to contact their PCP prior to visiting the ER.

\section{Experimental Design or Project Methods:}

This was a prospective, convenience survey, of low acuity ED patients from two academic, level 1 trauma centers in Indianapolis. After providing verbal consent, eligible patients were prompted by research staff, who verbally administered the survey and recorded the answers. Data were entered into a secure, HIPAA compliant database [REDCap] and the study was approved by the IRB. Demographic questions included patient's physician(s), mode of transportation to the ER, income, and habitation status. Baseline characteristics are analyzed with the statistical program SAS, reporting frequencies and proportions.

\section{Results:}

Of the 188 ER patients approached for the study, $144(77.4 \%)$ participated. $49.3 \%$ of participants were under the age of 50 , and $94(65.3 \%)$ were female. 49 (34.5\%) reported being racially white, with 86 (59.7\%) declaring to be African American. Over $2 / 3$ rds of these patients, 72 , had a yearly household income of 50,000 or less. $102(70.8 \%)$ patients affirmed having a PCP, with 61 (59.8\%) of that group reporting annual PCP visits. In the group stating annual PCP visits, only $23(38 \%)$ attempted to contact their physician before arriving at the ED.

\section{Conclusion:}

Based from our survey, it appears that our study population shows a lack of utilization of their PCPs, particularly lower acuity patients, before utilizing the ER. Future work could be done to better identity reasons and trends behind this. 